\title{
A Análise das Tendências Pluviométricas no Município de Irati/PR
}

\author{
Gabriela Marques Pinheiro ${ }^{(a)}$, Javier Martin-Vide ${ }^{(b)}$ \\ (a) Departamento de Geografia/ Universidade Estadual do Centro-Oeste, gabimpinheiro20@hotmail.com \\ (b) Departamento de Geografia Física e Análise Geográfica Regional /Universidade de Barcelona, Espanha, \\ j.martin.vide@hotmail.com
}

\section{Eixo: Climatologia em Diferentes Níveis Escalares: Mudanças e Variabilidades}

\begin{abstract}
Resumo
As mudanças climáticas globais, suas causas e consequências têm ganhado cada vez mais importância no cenário mundial. De acordo com o Primeiro Relatório de Avaliação Nacional (RAN-1), do Painel Brasileiro de Mudanças Climáticas, a projeção para mudanças da precipitação para a região Sul da América do Sul prevê, até 2040, aumento de 5\% a 10\%. Neste contexto, esta trabalho tem por objetivo principal compreender a variabilidade pluviométrica (1980 a 2010), assim como analisar tendências futuras para eventos pluviais no município de Irati, localizado na região sudeste do estado do Paraná. Dessa forma, foram utilizadas técnicas estatísticas para cálculo da variabilidade e tendências pluviométricas. Os resultados demostraram que a cidade não apresenta tendências positiva e negativa de precipitação para as últimas 30 décadas. Dessa forma, os dados corroboram para o melhor entendimento da gênese das chuvas bem como para os estudos sobre os impactos regionais e locais das mudanças climáticas e variabilidade climática.
\end{abstract}

Palavras chave: Mudanças Climáticas, Variabilidade Climática, Tendências Pluviométricas, Paraná, Irati

\section{Introdução:}

O conhecimento detalhado do clima e dos eventos climáticos extremos vem despertando interesse na sociedade atual, seja por este possuir caráter altamente dinâmico e apresentar rompimento na variabilidade natural ou apenas por estabelecer previsão de futuras mudanças do sistema climático. $\mathrm{O}$ estudo e o conhecimento atual do clima e sua variabilidade é um passo prévio e fundamental para a compreensão das mudanças climáticas por ação direta ou indireta do homem. Dificilmente pode-se falar sobre mudanças climáticas sem o conhecimento do clima atual.

Para o entendimento do clima, muitas variáveis são consideradas, como os subsistemas atmosféricos, hidrosféricos, litosféricos e biosféricos, os quais interagem de forma não linear com diferentes escalas de tempo, que vão desde dias (tempo atmosférico) até milhões de anos (processos geológicos), assim como diferentes escalas espaciais que vão desde metros (micrometeorologia) até a escala planetária (HERREZUELO, 2003). Dentro destas possibilidades de variáveis e escalas que permitem descrever o 
clima, o estudo da precipitação em escala diária torna-se o objeto central, dada a importância que este tem no Brasil e que apresenta grande variabilidade nas precipitações somando-se aos desequilíbrios hídricos.

Desde uma visão particular, nota-se que a precipitação no Brasil é muito irregular, devido a sua extensão territorial e às inúmeras massas de ar que atuam em todo o território, além de apresentar inúmeros fatores que contribuem para esta instabilidade, como a diversidade orográfica com importantes cadeias de montanhas, vales e rios, tornando, assim, os fenômenos locais únicos, os quais adquirem uma importância extraordinária. Estas condicionantes fazem com que as precipitações, sobre a escala diária, apresentem uma variabilidade tanto espacial quanto temporal, dificultando, assim, o seu entendimento.

Esta variabilidade da precipitação também se traduz em uma variabilidade hidrológica, ao passo que a irregularidade do regime hídrico no espaço e no tempo torna-se um dos tópicos mais discutidos, o qual se traduz na falta ou excesso de água ao longo dos anos. Entender a variabilidade climática e sua produção no espaço geográfico é fundamental para a implementação de ações que visão mitigar problemas que afetam diretamente ou indiretamente o cotidiano das pessoas e as atividades econômicas, como a agricultura, comércio, indústrias, produção de energia, abastecimento doméstico, turismo e meio ambiente.

As investigações científicas de caráter climatológico buscam entender e ultrapassar a quantificação da variabilidade dos elementos climáticos os quais, pautados na matemática-estatística, leva a considerar que estes, além de serem, produtos da intensidade, velocidade e frequência, são resultados da produção desigual do espaço geográfico em níveis de vulnerabilidade ao ritmo climático. Recentemente, estudos de Trenberth (2003) relacionam as mudanças climáticas às mudanças no clima local, relacionados à sua tendência climática.

As mudanças climáticas globais, suas causas e consequências, têm ganhado cada vez mais importância no cenário mundial, seja por sua visibilidade política, econômica, ambiental ou até mesmo pela criação de um cenário de preocupações, inquietações e alarmismo, de dimensão planetária, em face das possíveis repercussões deste processo em futuro próximo.

Mudanças climáticas podem ser resultantes naturais do sistema climático e sua variabilidade ou ser consequência de forte participação antropogênica; estas últimas são as mais importantes no processo, segundo o IPCC, 2007 (InternationalPanelonClimateChange). As causas de origem antropogênica estão associadas à influência das atividades humanas sobre o meio ambiente. Das mudanças produzidas ou provocadas pelo homem, tem merecido destaque a elevação da temperatura média no planeta e as mudanças nos padrões pluviométricos globais. 
XVII Simpósio Brasileiro

de Geografia Física Aplicada

I Congresso Nacional

de Geografia Física
OS DESAFIOS DA GEOGRAFIA FÍSICA NA FRONTEIRA DO CONHECIMENTO

Instituto de Geociências - Unicamp

Campinas - SP

28 de Junho à 02 de Julho de 2017

De acordo com RAN-1 (Relatório de Avaliação Nacional/Bases Cientificas), do Painel Brasileiro de Mudanças Climáticas (2014), a projeção para mudanças da temperatura e precipitação para a ampla região Sul da América do Sul prevê, até 2040, aumento relativamente baixo de temperatura entre $0,5^{\circ}$ e $1^{\circ} \mathrm{C}$ com um aumento de 5\% a 10\% na chuva. Em meados do século (2041-2070), mantêm-se as tendências de aumento gradual de $1,5^{\circ}$ a $2^{\circ} \mathrm{C}$ na temperatura e aumento de $15 \%$ a $20 \%$ nas chuvas, sendo que essas tendências acentuam-se ainda mais no final do século (2071-2100), com padrões de clima entre $2,5^{\circ}$ e $3^{\circ} \mathrm{C}$ mais quente e entre $25 \%$ a $30 \%$ mais chuvoso.

No Brasil, devido à grande extensão territorial, encontramos diferentes dinâmicas pluviométricas, influenciadas pelas características físicas do território, pela dinâmica atmosférica atuante e pela variação latitudinal. Diferentes regimes de precipitação são observados no Brasil, sendo que a sua distribuição espacial e temporal (diária, mensal e anual) deriva efeitos diretos e indiretos no espaço. Assim, nesta pesquisa, a área de estudo limitou-se ao estado do Paraná, mais precisamente ao município de Irati/PR.

\section{Contextualização:}

Segundo as perspectivas do IPCC (InternationalPanelonClimateChange), as áreas urbanas tendem a concentração populacional, gerando graves problemas sociais e ambientais de todas as classes. Enquanto os riscos e vulnerabilidades, perante os problemas ambientais urbanos, tendem a intensificar, os estudos os quais permitam os enfrentamentos, mitigações e adaptações, perante as inundações urbanas, é necessário.

Os eventos climáticos extremos de precipitação estão entre as causas deflagradoras de desastres naturais que afetam a sociedade. Os regimes das chuvas e sua variabilidade natural são afetados pelas transformações da superfície da terra, passando por períodos irregulares, marcados por períodos chuvosos e secos, quentes e frios, responsáveis por inúmeras repercussões espaciais.

Desta forma, os fenômenos associados com as flutuações pluviométricas, como episódios de enchentes e inundações, são constantes, principalmente nas grandes cidades do Sul e Sudeste do Brasil. Os eventos de inundações apresentaram-se críticos entre os anos de 1974 a 2003 e, segundo dados EM-DAT (banco de dados de eventos de emergência) as catástrofes naturais vem aumentando, entre os anos de 1948 e 2007.

De acordo com RAN-1 (Relatório de Avaliação Nacional/Bases Cientificas), do Painel Brasileiro de Mudanças Climáticas (2014), a projeção para mudanças na precipitação para a ampla região Sul da América do Sul prevê, até 2040, aumento de 5\% a 10\% na chuva. Em meados do século (2041-2070), mantêm-se as tendências de aumento gradual de $15 \%$ a $20 \%$ nas chuvas, sendo que essas tendências 


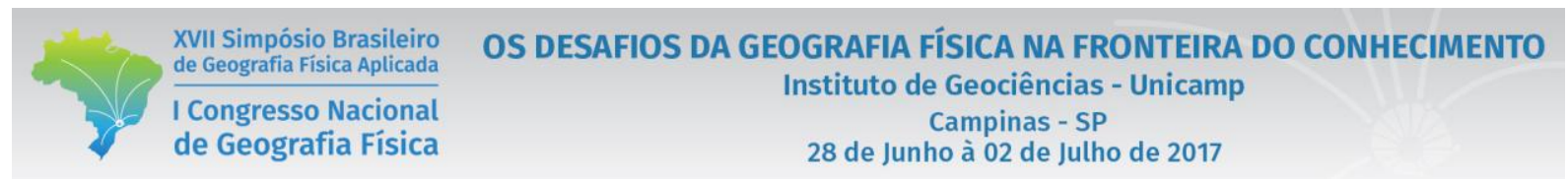

acentuam-se ainda mais no final do século (2071-2100), com padrões de clima entre $25 \%$ a $30 \%$ mais chuvoso.

Segundo as hipótesesvinculadas pelo IPCC, a Região Sul do Brasil será afetada futuramente com a ocorrência de cenários de climas mais extremos, com ênfase para o aumento da frequência de eventos extremos ocasionando maior ocorrência de inundações e alagamentos. Além do volume pluviométrico, a vulnerabilidade dos municípios da região contribui para a gravidade do evento.

Dentro deste contexto, a pluviosidade é uma das mais importantes variáveis meteorológicas para os estudos climáticos. Essa importância deve-se a sua função dentro da dinâmica climática e de sua variação no espaço e no tempo. Além disso, ela constitui uma das variáveis climáticas que mais influência no cotidiano das pessoas e na qualidade do meio ambiente. Os volumes de precipitação pluvial, a distribuição temporal e a intensidade das chuvas afetam a sociedade, a economia e a dinâmica natural do planeta.

Desta maneira, a pesquisa tem como objetivo compreender as possíveis tendências pluviométricas, da região sudeste do Paraná, mais precisamente o município de Irati, Figura 01.

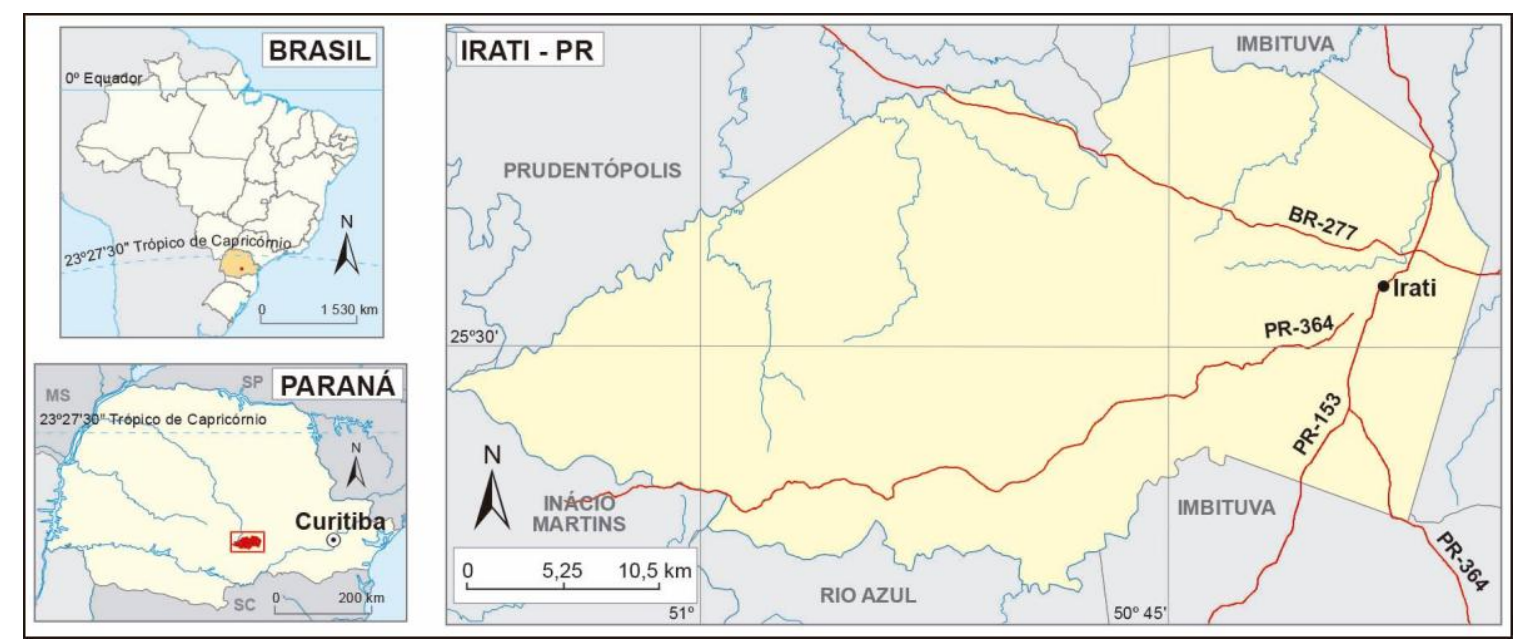

Figura 01 - Localização da área de estudo

A área a ser estudada possui, segundo o IBGE (2013), um total de 59.030 mil habitantes, ocupando uma área total de $999,515 \mathrm{Km}^{2}$, com uma densidade média de 59,06 hab./ $\mathrm{Km}^{2}$ e a altitude é de 812 . A região encontra-se inserida no contexto global, o qual constitui parte do cenário das mudanças climáticas globais e participa, portanto, do jogo de causas e consequências do processo. A interação entre a dimensão 
XVII Simpósio Brasileiro

de Geografia Física Aplicada

I Congresso Nacional

de Geografia Física
OS DESAFIOS DA GEOGRAFIA FÍSICA NA FRONTEIRA DO CONHECIMENTO

Instituto de Geociências - Unicamp

Campinas - SP

28 de Junho à 02 de Julho de 2017

regional e a dimensão global do fenômeno constitui o centro da análise no que concerne a variabilidade pluviométrica. Dessa forma, o foco principal de análise remete ao estudo da variabilidade pluviométrica e sua repercussão espaço-temporal, associando abordagens qualitativa (dinâmica) e quantitativa (estatística).

A região sudeste do estado do Paraná, mais precisamente o município de Irati foi definida como área de estudo, pois vem experimentando o processo de crescimento da população nas últimas décadas, associada a problemas de ordens socioambientais, como frequentes inundações urbanas.

\section{Problema:}

Os eventos naturais são registrados com frequência e, portanto, a sociedade se estruturaria de forma a absorvê-los, adaptando-se ao seu ritmo e considerando-os como normais ou habituais. Os chamados fenômenos normais - aqueles que não se afastam significativamente da média - ocorrem em alta frequência e cotidianamente (GONÇALVES, 2003). Já os eventos extremos, segundo Sarewitz e Pielke Jr. (2000), são definidos pela ocorrência e apresentação em uma incidência rara, distanciando-se da média e variando em sua magnitude. Os eventos extremos de chuva podem ser considerados aqueles cujos totais anual, sazonal ou diário demonstram desvios de valores superiores ou inferiores à apresentação dos habituais da área no período analisado.

Diante desta situação, os eventos climáticos extremos apresentam-se entre as causas deflagradoras de catástrofes naturais que atingem a sociedade. Os regimes pluviométricos e térmicos, além de sua variabilidade natural, são os mais afetados pelas transformações da superfície terrestre, passando a exercer comportamentos irregulares, marcados por alternâncias de períodos chuvosos e secos, quentes e frio, responsáveis por inúmeras repercussões de ordem calamitosas sobre os mais variados espaços geográficos (SILVA, 2007).

Dessa forma, a partir dos conhecimentos bibliográficos gerados, nota-se a possiblidade de classificações e denominação de eventos extremos a partir de sua natureza, sendo subdivido entre geográficos e meteorológicos/climáticos. Os eventos de natureza geográfica se referem às enchentes, aos deslizamentos, aos movimentos de massa, principalmente em áreas ocupadas por atividades humanas, pois possuem relação direta com produção do espaço geográfico. Nesses tipos de eventos inserem-se as tragédias, os desastres e a ocorrência de mortes.

Os de natureza meteorológica e climática aludem à ocorrência de, por exemplo, furacões, chuvas intensas e rápidas, secas, que de forma geral são considerados no contexto natural da variabilidade e das variações do sistema climático e meteorológico. 
XVII Simpósio Brasileiro

de Geografia Física Aplicada

I Congresso Nacional

de Geografia Física
OS DESAFIOS DA GEOGRAFIA FÍSICA NA FRONTEIRA DO CONHECIMENTO

Instituto de Geociências - Unicamp

Campinas - SP

28 de Junho à 02 de Julho de 2017

Dentre estas manifestações no Brasil, os fenômenos relacionados às oscilações hídricas e os episódios pluviais extremos de ordem negativa (enchentes/inundações) são os mais significantes nas cidades e possuem o caráter de calamidade a partir do momento em que causam impactos no meio ambiente, bem como na vida social e econômica do pais.

Segundo Monteiro (1991):

[...] uma concepção mais comportamental e complexa do clima é que nos damos contade como os estados de tempo oscilam, admitem desvios e produzem 'acidentes' que não podem ser considerados excepcionais. Mesmo os mais graves, por vezes catastróficos, se estatisticamente poderiam ser tomados como 'ruído', seu impacto local ou regional e a reação em cadeia que se lhes segue, fazem com que eles não possam ser descartados. E,

sobretudo por suas repercussões socioeconômicas, tanto por injúrias imediatas como pelo efeito de 'desregularização' da produção agrícola, do sistema hidro-energético, da rede de transportes, etc... (MONTEIRO, 1991, p. 19).

Sendo assim, dentro do atual contexto de aquecimento global, são esperados eventos de chuvas mais intensos e mais frequentes, como resultado do maior conteúdo de água na atmosfera, favorecido pela maior instabilidade termodinâmica (KUNKEL, 2003). Seguindo este raciocínio, o quarto relatório do IPCC mostra que houve aumento de ocorrência de chuvas intensas em muitas regiões, em latitudes médias, durante o período de 1951 a 2003. Estas ocorrências eram esperadas, em razão das mudanças na quantidade total de chuvas (IPCC, 2007). Porém, atenta-se que, na escala regional, essas mudanças são muito divergentes e nem sempre acompanham a tendência global. Apesar da grande ressalva que se faz atualmente

A partir destes parâmetros, entender as tendências pluviométricas caracteriza-se como um dos fios condutores da abordagem adotada neste estudo. Nesta perspectiva, o espaço geográfico é o receptáculo do ritmo climático e depositário final e obrigatório, revelando as repercussões na forma de impacto.

Nota-se, de acordo com trabalhos de Minikowski e Maia, que as inundações representam um cenário critico no município de Irati entre os anos de 1983 a 2010 e que elas estão associadas a eventos normais e extremos de precipitação no município. Desta forma, este trabalho corroborou para o entendimento da dinâmicas pluviométricas, as quais segundo o IPCC, tendem a crescer nos próximos décadas. Sendo assim, esta pesquisa poderá colaborar com os futuros planos diretores assim como a efetivação de políticas voltada a doenças de cunho hídrico. 


\section{Materiais e Método:}

Segundo Yevjevich (1972) tendência pode ser definida como uma série temporal, como uma mudança sistemática e contínua, em qualquer parâmetro de uma dada amostra, excluindo-se mudanças periódicas ou quase periódicas.

Já segundo Goossense Berger (1986), flutuação climática é qualquer forma de mudança sistemática regular ou irregular, exceto a tendência e mudança abrupta. Oscilação climática é a flutuação na qual a variável tende a mover-se gradualmente e de forma suave entre sucessivos máximos e mínimos. A tendência climática é uma mudança climática caracterizada por um suave acréscimo ou decréscimo nos valores médios no período de registro. A mudança climática abrupta é uma mudança abrupta e permanente durante o período de registro de um valor médio para outro.

Sendo assim, o modelo do Excel "Makesens" (SALMI et al. 2002) foi pensando para atender à execução do teste de Mann-Kendall (MANN, 1945; KENDALL, 1975). A planilha Makesens foi desenvolvida para detectar e estimar tendência em série histórica suficientemente longa de valores anuais de concentrações atmosféricas e precipitações. Por meio desta, se executam dois tipos de análises estatísticas. Primeiro, é verificada a existência de tendência monotônica, que pode ser crescente ou decrescente, através do teste não paramétrico de Mann-Kendall. Em seguida, é examinada a inclinação da tendência linear, que é estimada através do método não paramétrico “Sen”, conforme explica Gilbert (1987).

Em uma série de variável climática, como a precipitação pluvial, pode ocorrer variações significativas ou não. O procedimento estatístico que analisa a existência de alterações é denominado de análise de regressão, onde são exibidas as representações dos valores com irregularidades do padrão, referentes ao período analisado. Clark e Hosking (1986) explicam que é pouco provável que as séries climáticas possuam tendências lineares. Por isso, se usa o método não paramétrico. Para Benhamrouche (2014), os modelos paramétricos têm limitações, pois admitem homogeneidade na variação ao longo das séries de chuvas que não correspondem à realidade da variável. Com a finalidade de não ser descartada nenhuma série histórica, o referido autor recorda que estas necessitam apresentar longitude temporal suficientemente longa.

O teste de Mann-Kendall (MANN, 1945; KENDALL, 1975) foi usado para alcançar os resultados coerentes quando aplicado a área de estudo. Os cálculos são efetuados através da planilha Makesens versão 1.0 desenvolvida por Tino Salmi do Instituto Meteorológico da Finlândia (SALMI et al., 2002), por meio da ferramenta Microsoft Excel 2010 e a macro do Microsoft Visual Basic. É avaliada a convergência linear crescente ou decrescente, ou seja, verifica-se a inclinação da reta e o grau de significância juntamente com a estimação "Sen" (SEN, 1968) da pendente para completar o teste de Mann-Kendall. 
Para tanto, foram utilizados dados diários posteriormente convertidos em dados mensais para o período de 30 anos (1980 a 2010). Os dados foram separados por estações do ano como primavera, verão, outono e inverno, com o intuito de realizar analises sazonais, pois sabe-se que oscilações influenciam e variações são encontradas ao longo destes meses, além de que sistemas atmosféricos atuantes em cada uma destas estações são determinantes para a coleta dos dados. A estação meteorológica utilizada foi do município de Irati, localizada no Colégio Agrícola. Estes métodos de estudos estão recomendados pela OMM, sobretudo quando a variável climática analisada é a chuva.

A existência ou não de tendência, será baseado nos níveis de significância expostos na tabela I. É importante a valorização dos graus de significância estatística dos resultados, já que quanto mais alto é o grau de significância, mais fiel ou confiável eles podem ser; no mesmo sentido é a tendência.

Tabela I - Nível de Significância, símbolos e significância, teste de Mann Kendall

\begin{tabular}{ccc}
\hline Níveis de Significância & Símbolos & Significância \\
Excelente & $* * *$ & $99,9 \%$ \\
Muito Boa & $* *$ & $99 \%$ \\
Boa & $*$ & $95 \%$ \\
Aceitável & + & $90 \%$ \\
Duvidosa & Em branco & Menor que 90\% \\
\hline
\end{tabular}

\section{$5 \quad$ Resultados}

A análise de tendência foi realizada para a estações meteorológica localizada na cidade de Irati, precisamente inserida no Colégio Agrícola. Dessa forma, foi utilizado o teste de Mann-Kendall para verificar possíveis tendências, tanto positiva, quanto negativa, ou mesmo nula. Primeiramente, foi realizado o teste para o total anual e, seguidamente, para os totais sazonais.

O teste (Tabela II) revelou que nenhuma estação apresentou tendência positiva, com significância acima de $90 \%$.

Tabela II - Teste Estatístico de Mann-Kendall para o Total Anual (mm)

\begin{tabular}{|c|c|c|c|c|c|c|}
\hline Time series & Firstyear & $\begin{array}{c}\text { Last } \\
\text { Year }\end{array}$ & N & $\begin{array}{c}\text { Test } \\
\text { S }\end{array}$ & Test Z & Significância. \\
\hline 0 & 1980 & 2010 & 31 & & 0,27 & \\
\hline
\end{tabular}

A análise sazonal, dentro do contexto da variabilidade climática, é fundamental para compreender a dinâmica em escala regional que em muitos casos divergem e nem sempre acompanham a tendência global. A visualização dos totais das variáveis selecionadas corresponde à análise exploratória do padrão temporal dos dados de precipitação. Na tabela III está apresentado o cálculo da estatística Sazonal de Mann-Kendall para a Primavera para verificar se há tendência estatisticamente significativa nas séries 
XVII Simpósio Brasileiro

de Geografia Fisica Aplicada

I Congresso Nacional de Geografia Física
OS DESAFIOS DA GEOGRAFIA FÍSICA NA FRONTEIRA DO CONHECIMENTO

Instituto de Geociências - Unicamp

Campinas - SP

28 de Junho à 02 de Julho de 2017

temporais de dados de precipitação registrados no posto meteorológico. Nota-se que a primavera (Tabela III) não apresentou tendência positiva e nem negativa, desta forma os resultados foram nulos, assim como o verão (Tabela IV), outono (Tabela V) e inverno (Tabela VI).

Tabela III - Teste Estatístico de Mann-Kendall para Primavera (mm)

\begin{tabular}{|c|c|c|c|c|c|c|}
\hline Time series & Firstyear & $\begin{array}{c}\text { Last } \\
\text { Year }\end{array}$ & N & $\begin{array}{c}\text { Test } \\
\text { S }\end{array}$ & Test Z & Significância. \\
\hline 0 & 1980 & 2010 & 31 & & 1,09 & \\
\hline
\end{tabular}

Tabela IV - Teste Estatístico de Mann-Kendall para o Verão (mm)

\begin{tabular}{|c|c|c|c|c|c|c|}
\hline & Last & Test & & \\
Time series & Firstyear & Year & N & S & Test Z & Significância. \\
\hline 0 & 1980 & 2010 & 31 & & 0,41 & \\
\hline
\end{tabular}

Tabela V - Teste Estatístico de Mann-Kendall para o Outono (mm)

\begin{tabular}{|c|c|c|c|c|c|c|}
\hline Time series & Firstyear & $\begin{array}{l}\text { Last } \\
\text { Year }\end{array}$ & $\mathbf{N}$ & $\begin{array}{c}\text { Test } \\
\text { S }\end{array}$ & Test $\mathrm{Z}$ & Significância. \\
\hline $\mathbf{0}$ & 1980 & 2010 & 31 & & $-0,31$ & \\
\hline
\end{tabular}

Tabela VI - Teste Estatístico de Mann-Kendall para o Inverno (mm)

\begin{tabular}{|c|c|c|c|c|c|c|}
\hline Time series & Firstyear & $\begin{array}{c}\text { Last } \\
\text { Year }\end{array}$ & N & $\begin{array}{c}\text { Test } \\
\text { S }\end{array}$ & Test Z & Significância. \\
\hline 0 & 1980 & 2010 & 31 & & $-0,61$ & \\
\hline
\end{tabular}

\section{Conclusões e Considerações Finais}

Dessa forma, conclui-se, para a análise de tendência dos totais anuais e sazonais, que nenhuma estação do ano, assim como os totais, apresentaram tendências significativas de crescimento das chuvas ao longo destes 31 anos de análise.

Sendo assim, a pesquisa conclui que o município de Irati, não apresenta conformidade com as informações expostas pelo IPCC, o qual sugere o aumento nos totais de precipitação. É sabido que o período considerado para a análise é pequeno em consideração às escalas trabalhadas pelas mudanças climáticas. Dessa forma, os resultados foram trabalhados em conformidade de conceito, acreditando que a área de estudo apresenta uma variabilidade natural, a qual está sujeita a variações no ritmo climático.

No geral, os resultados corroboram para o melhor entendimento da gênese das chuvas. $\mathrm{O}$ estudo teve como objetivo colaborar com a avaliação dos impactos regionais e locais perante os eventos de mudanças climáticas e possíveis estudos de análise dos desastres associados ao aumento da precipitação associados aos estudos de variabilidade climática. 


\section{Referências:}

BENHAMROUCHE, A. Análisis de laconcentracióndiaria de laprecipitaciónenla Cuenca delMediterráneoOccidental. Tesis (DoctoradoenGeografía). Programa de DoctoradoenGeografía, Planificación Territorial y Gestión Ambiental da Universidad de Barcelona, Barcelona-España. 2014 Disponível em: . Acesso em: 28 mar. 2015.

CLARK, W. A. V. and HOSKING, P. L.: Statisticalmethods for geographers. New York: John Wiley\& Sons, Inc. 1986.

EM-DAT, EmergencyEventsDatabase. The OFDA/CRED InternationalDisasterDatabase. Disponível em: Acesso em dezembro de 2016.

EM-DAT. EmergencyEventsDatabase. Annualdisasterstatisticalreview. 2012.

GILBERT, R. O. Statisticalmethods for environmentalpollutionmonitoring. New York: Van Nostrand Reinhold, 1987.

GONÇALVES, N. M. S. Impactos Pluviais e Desorganização do Espaço Urbano em Salvador. In: MONTEIRO, C. A. de F.; MENDONÇA, F. Clima Urbano. São Paulo: Contexto, 2003. p. 69-92.

GOOSSENS, C.; BERGER, A. Annual and seasonalclimaticvariationsoverthenorthemhemisphere and Europeduringthelastcentury. AnnalesGeophysicae, Berlin, v.4, 1986.

GRIMM, A. M. Variabilidade interanual do clima do Brasil. In: CAVALCANTI, I, F. A; FERREIRA, N. J; SILVA, M, G, A, J; DIAS, M. A. F. S (Orgs). Tempo e clima no Brasil. Oficina de Textos. 2009.

HERREZUELlo, M. da C. G. Un Estudio de la Variabilidad Climática en la Península Ibérica. 344f. Tese de Doutorado em Ciências Físicas, Universidad de Extremadura. 2003.

IBGE. Instituto Brasileiro de Geografia e Estatística. Disponível em: http://www.ibge.gov.br/home/estatistica/populacao/censo2010. Acesso em: novembro 2014.

IPCC, 2007: ClimateChange 2007: The Physical Science Basis. ContributionofWorkingGroup I totheFourthAssessmentReportoftheIntergovernmentalPanelonClimateChange [Solomon, S., D. Qin, M. Manning, Z. Chen, M. Marquis, K.B. Averyt, M.Tignorand H.L. Miller (eds.)]. Cambridge University Press, Cambridge, United Kingdomand New York, NY, USA.

KENDALL, M. G. Rankcorrelationmethods. 4a ed., London: Charles Griffin, 1975.

LIMA, R.; INOUE, M. T.; FIGUEIREDO FILHO, A.; ARAUJO, A. J.; MACHADO, S. A. Efeito do espaçamento no desenvolvimento volumétrico de Pinus taeda L. Floresta e Ambiente, n. 20, v. 2, p. 223-230, 2013.

MANN, H. B. Nonparametrictestsagainsttrend. Econometrica, vol. 13, n. 3, 1945

MENDONÇA, F.; DANNI-OLIVEIRA, I. M.; Climatologia: noções básicas e climas do Brasil. São Paulo. Oficina de texto. 2007.

MONTEIRO, C. A. F. O clima da região Sul. Clima e excepcionalismo: conjecturas sobre o desempenho da atmosfera como fenômeno geográfico. Florianópolis: Editora da UFSC, 1991.

OMM/ ETCCDMI. World MeteorologicalOrganization - The Expert Team onClimateChangeDetectionMonitoringandIndices: GuidelinesonAnalysisof extremes in a changingclimate in supportofinformeddecisions for adaptation. 2009.

p. 245- 259 p. The EconometricSociety. 
SALMI, T.; MÄÄTTÄ, A.; ANTTILA, P.; RUOHO-AIROLA, T.; AMNELL, T. Makesens for detectingandestimatingtrends. Helsinki: FinnishMeteorologicalInstitute, 2002.

SALMI, T.; MÄ̈̈TTÄ, A.; ANTTILA, P.; RUOHO-AIROLA, T.; AMNELL, T. Makesens for detecting and estimating trends.Helsinki: FinnishMeteorologicalInstitute, 2002.

SEN, P. K. EstimatesoftheregressioncoefficientbasedonKendall's tau. Journalofthe American StatisticalAssociation, vol. 63, n. 324, 1379-1389 p. 1968.

SILVA, E.S. Variabilidade da precipitação pluviométrica nas regiões sudeste e sul do Brasil. $211 \mathrm{f}$. Tese (Doutorado em Agronomia) - Faculdade de Ciências Agronômicas da UNESP - Campus de Botucatu, Universidade Estadual de São Paulo. Botucatu, 2007.

SILVA, I. F.; NÓBREGA, R. S.; GALVÍNCIO, J. G. Impacto das Mudanças Climáticas nas Respostas Hidrológicas do Rio Ipojuca (PE) - Parte 2: Cenários de Aumento de Temperatura. Revista Brasileira de Geografia Física (RBGF). Recife-PE, Vol.2 (maio-agosto 2009). n.02.

TRENBERTH, K. E.; A. DAI, R. M. Rasmussen and D. B. Parsons. 2003. The changing character of precipitation. BAMS 84(9):1205-1217.

YEVJEVICH, V. Probability and statistics in hydrology. Fort Collins : Water Resources Publication, p. 276. 1972. 